\title{
Monitoramento de perdas quantitativas no recolhimento de amendoim
}

Submetido - 31 jul. $2020 \quad$ Aprovado - 04 set. $2020 \quad$ Publicado - 14 out. 2020
do ${ }_{\text {http://dx.doi.org/10.17648/sas.v1i1.79 }}$

Armando Lopes de Brito Filho (D)

Mestrando do Programa de Pós-Graduação em Agronomia (Ciência do Solo), Universidade Estadual Paulista, Campus Jaboticabal, e-mail: armando.brito@unesp.br.

\section{Jarlyson Brunno Costa Souza iD}

Mestrando do Programa de Pós-Graduação em Agronomia (Produção Vegetal), Universidade Estadual Paulista, Campus Jaboticabal, e-mail: jarlyson.brunno@unesp.br.

\section{Samira Luns Hatum de Almeida iD}

Doutoranda do Programa de Pós-Graduação em Agronomia (Produção Vegetal), Universidade Estadual Paulista, Campus Jaboticabal, e-mail: samira.Ih.almeida@unesp.br.

Tulio Mazetti Marra ${ }^{(D)}$

Engenheiro de Biossistemas, Universidade de São Paulo, e-mail: tulio.marra@usp.br.

Franciele Morlin Carneiro (D)

Pós-Doutoranda do Programa de Pós-Graduação em Agronomia (Produção Vegetal), Universidade Estadual Paulista, Campus Jaboticabal, e-mail: franciele.morlin@unesp.br.

Prof. Dr. Livre-Docente da UNESP/FCAV (Produção Vegetal), Jaboticabal-SP, e-mail: rouverson.silva@unesp.br.

\section{RESUMO}

Dentre as operações agrícolas, a colheita mecanizada é a etapa final que merece muita atenção por afetar diretamente na produtividade, ou seja, quanto maior for a quantidade de perdas haverá reduções de produtividade e o aumento de custos. Assim análises estatísticas como o Controle Estatístico de Qualidade (CEQ) está sendo aplicado na agricultura e tem demonstrado grande potencial para a melhoria da gestão dos sistemas agrícola bem como nas tomadas de decisão. Com este trabalho, objetivou-se monitorar a qualidade operacional da colheita mecanizada do amendoim, durante o recolhimento, por meio do $C E Q$, e quantificar as perdas totais com a utilização da armação retangular. 0 experimento foi realizado, em área comercial, na safra 2019/2020, no município de Ribeirão Preto, estado de São Paulo, localizado nas coordenadas geográficas $21^{\circ} 20^{\prime} 17.55^{\prime \prime S}$ e $47^{\circ} 54^{\prime} 7.31^{\prime \prime O}$. O amendoim foi semeado em sistema de Meiosi (Método Inter Ocupacional Simultâneo). $O$ delineamento experimental seguiu as premissas do CEQ, monitorando, ao longo do tempo, 20 pontos amostrais que foram distanciados entre si com $80 \mathrm{~m}$ de comprimento. 0 indicador de qualidade avaliado, durante 0 recolhimento, foram as perdas totais que foram quantificadas por meio da armação retangular, possuindo as seguintes dimensões $5,4 \mathrm{~m}$ de largura por $0,37 \mathrm{~m}$ de comprimento. A análise estatística foi executada por meio das ferramentas do CEQ, que foram: cartas de controle de valores individuais, gráficos sequenciais ou run charts e análise descritiva. Concluiu-se que por meio da aplicação das ferramentas de qualidade permitiu o maior acompanhamento e monitoramento da operação, em que não houve presença de causas especiais e nem de padrões de não aleatoriedade.

Palavras-chave: Arachis hypogaea L.; Cartas de controle; Colheita mecanizada.

\section{Monitoring quantitative losses in peanut harvesting}

\section{ABSTRACT}

Among agricultural operations, mechanized harvesting is the final step that deserves a lot of attention because it directly affects productivity, that is, the greater the amount of losses, there 
will be reduced productivity and increased costs. Thus, statistical analyzes such as the Statistical Quality Control (CEQ) are being applied in agriculture and have shown great potential for improving the management of agricultural systems as well as in decision making. With this work, the objective was to monitor the operational quality of mechanized peanut harvesting, during collection, through CEQ, and to quantify the total losses with the use of the rectangular frame. The experiment was carried out, in a commercial area, in the 2019/2020 harvest, in the municipality of Ribeirão Preto, state of São Paulo, located at geographic coordinates $21^{\circ} 20^{\prime} 17.55$ "S and 47 ${ }^{\circ} 54^{\prime} 7.31^{\prime \prime} O$. The peanut was sown in a Meiosi system (Simultaneous Inter-Occupational Method). The experimental design followed the premises of the CEQ, monitoring, over time, 20 sample points that were $80 \mathrm{~m}$ long apart. The quality indicator evaluated, during the collection, was the total losses that were quantified through the rectangular frame, having the following dimensions $5.4 \mathrm{~m}$ wide by $0.37 \mathrm{~m}$ long. The statistical analysis was performed using the CEQ tools, which were: individual value control charts, sequential or run charts and descriptive analysis. It was concluded that, through the application of quality tools, it allowed greater monitoring and monitoring of the operation, in which there was no presence of special causes or patterns of non-randomness.

Keywords: Arachis hypogaea L.; Control charts; mechanized harvest.

\section{Monitoreo de pérdidas cuantitativas en la cosecha de maní}

\section{RESUMEN}

Entre las operaciones agrícolas, la cosecha mecanizada es el paso final que merece mucha atención porque afecta directamente la productividad, es decir, cuanto mayor sea la cantidad de pérdidas, habrá menor productividad y mayores costos. Por lo tanto, los análisis estadísticos como el Control Estadístico de Calidad (CEQ) se están aplicando en la agricultura y han demostrado un gran potencial para mejorar la gestión de los sistemas agrícolas, así como en la toma de decisiones. Con este trabajo, el objetivo era monitorear la calidad operativa de la cosecha mecanizada de maní, durante la recolección, a través de CEQ, y cuantificar las pérdidas totales con el uso del marco rectangular. El experimento se llevó a cabo, en un área comercial, en la cosecha 2019/2020, en el municipio de Ribeirão Preto, estado de São Paulo, ubicado en las coordenadas geográficas $21^{\circ} 20^{\prime} 17.55$ "S y $47^{\circ} 54 ' 7.31^{\prime \prime} O$. El maní se sembró en un sistema Meiosi (método simultáneo entre ocupaciones). El diseño experimental siguió las premisas del CEQ, monitoreando, con el tiempo, 20 puntos de muestra que estaban separados por $80 \mathrm{~m}$ de largo. El indicador de calidad evaluado, durante la recolección, fue las pérdidas totales que se cuantificaron a través del marco rectangular, teniendo las siguientes dimensiones $5.4 \mathrm{~m}$ de ancho por $0.37 \mathrm{~m}$ de largo. El análisis estadístico se realizó utilizando las herramientas $C E Q$, que fueron: gráficos de control de valor individual, gráficos secuenciales 0 de ejecución y análisis descriptivo. Se concluyó que, a través de la aplicación de herramientas de calidad, permitió un mayor monitoreo y monitoreo de la operación, en la cual no había presencia de causas especiales o patrones de no aleatoriedad.

Palabras clave: Arachis hypogaea L., Cuadros de control, Cosecha mecanizada.

\section{Introdução}

O maior produtor mundial de amendoim é a China e o Brasil é o segundo maior exportador da América Latina, com uma produção total de 466 mil toneladas no ano de 2019, no qual exportou $30 \%$ de sua produção, ficando atrás apenas da Argentina, que exportou quase $80 \%$ do seu montante de produção, de aproximadamente um milhão de toneladas (SANTOS, 2019). De acordo com o mesmo autor, o estado que concentra a maior produção desta cultura é São Paulo com mais de $90 \%$ da produção nacional do grão. 
Dentre as operações agrícolas que merecem bastante atenção é a colheita, pois é a etapa final do sistema agrícola, sendo que, quando realizada de forma inadequada vai haver maiores quantidade de perdas e isso afetará diretamente na produtividade. Visto que, a colheita de amendoim possui um grande diferencial entre várias culturas devido o desenvolvimento subterrâneo das vagens, tendo que aumentar mais ainda o cuidado ao longo manejo da colheita.

Estudos mostram que, na região da Alta Mogiana Paulista no início do século do século XXI, as perdas médias no arranquio do amendoim eram de 30\% (SILVA, 2019). De acordo com o mesmo autor, em boas condições climatológicas e com a produção recebendo um gerenciamento operacional adequado, as perdas médias podem ser mantidas próximas a $5 \%$.

Vendo a necessidade da melhoria dessa operação, o Controle Estatístico de Qualidade (CEQ) tem sido muito aplicado na agricultura, pois é um conjunto de ferramentas de qualidade que visa o monitoramento e controle de qualidade, conseguindo-se uma descrição detalhada do comportamento do processo estudado, identificando sua variabilidade e possibilitando a detecção de erros, responsáveis pelas instabilidades do processo em estudo (ALENCAR et al., 2005).

Objetivou-se, com este trabalho, monitorar a qualidade operacional da colheita mecanizada do amendoim por meio do CEQ, e quantificar as perdas totais com a utilização da armação retangular.

\section{Material e métodos}

O experimento foi realizado, em área comercial, na safra 2019/2020, no município de Ribeirão Preto, estado de São Paulo, localizado nas coordenadas geográficas $21^{\circ} 20^{\prime} 17.55^{\prime \prime S}$ e $47^{\circ} 54^{\prime} 7.31^{\prime \prime O}$. O amendoim foi semeado em sistema de Meiosi (Método Inter Ocupacional Simultâneo), onde há o cultivo do amendoim com a cana-de-açúcar. Em que foi distribuído 18 sementes $\mathrm{m}^{-1}$ da cultivar IAC OL3. A área experimental possui o solo Latossolo Vermelho e o clima da região é Aw, tropical com inverno seco, conforme a classificação climática de Köppen (ALVARES et al., 2013). 
O delineamento experimental seguiu as premissas do CEQ, monitorando, ao longo do tempo, 20 pontos amostrais que foram distanciados entre si com $80 \mathrm{~m}$ de comprimento. No recolhimento foi utilizado o trator John Deere 4x2 TDA, modelo $7195 \mathrm{~J}$, com potência de 143,5 kW (195 cv), que foi acoplado na recolhedora de marca Miac, modelo Twin Master Tripla, com largura da plataforma $5,40 \mathrm{~m}$ e capacidade de recolher 3 leiras (6 linhas) de amendoim. A velocidade de descolamento, média, foi de $3,8 \mathrm{~km} \mathrm{~h}^{-1}$.

$\mathrm{O}$ indicador de qualidade avaliado, durante o recolhimento, foram as perdas totais que foram quantificadas por meio da armação retangular, possuindo as seguintes dimensões $5,4 \mathrm{~m}$ de largura por $0,37 \mathrm{~m}$ de comprimento. A análise estatística foi executada por meio das ferramentas do $C E Q$, que foram: cartas de controle de valores individuais, gráficos sequenciais ou run charts e análise descritiva.

\section{Resultados e discussão}

Nas cartas de controle de valores individuais (Figura 1A.) e de amplitude móvel (Figura 1B.) foi observado que não houve pontos fora de controle, obtendo um processo estável em virtude não ter tido causas especiais que afetam no aumento de variabilidade. De acordo com Montgomery (2016) qualidade e variabilidade são inversamente proporcionais.

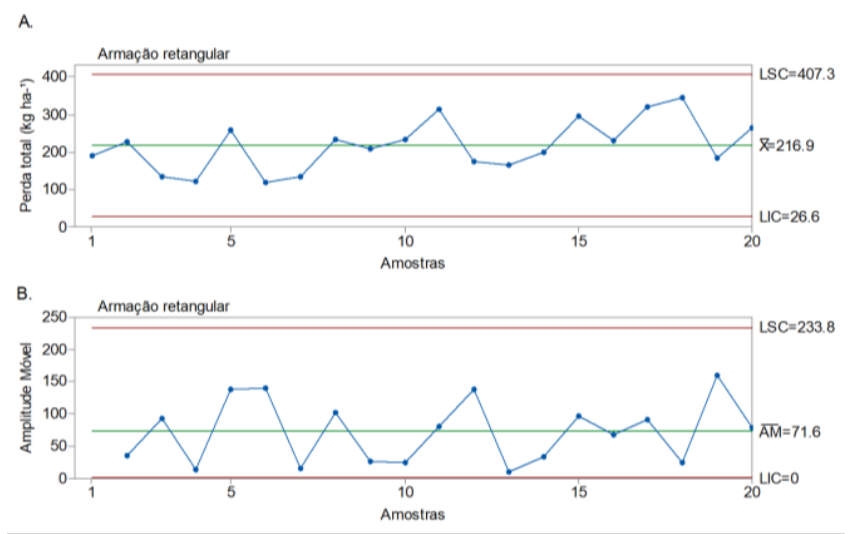

Figura 1. Cartas de controle de valores individuais (A.) e amplitude móvel (B.) para perdas totais $\left(\mathrm{kg} \mathrm{ha}^{-1}\right)$.

Nem sempre quando não há pontos fora de controle significa que não há padrões de não aleatoriedade, por isso é importante a 
utilização de ferramentas complementares com as cartas de controle como os gráficos sequenciais. Por isso, foi confeccionado o gráfico sequencial, para o maior entendimento do comportamento do processo. Foi verificado que não houve presença desses padrões, pois o p-valor foram maiores que 0,05 , como pode ser visto na Figura 2.

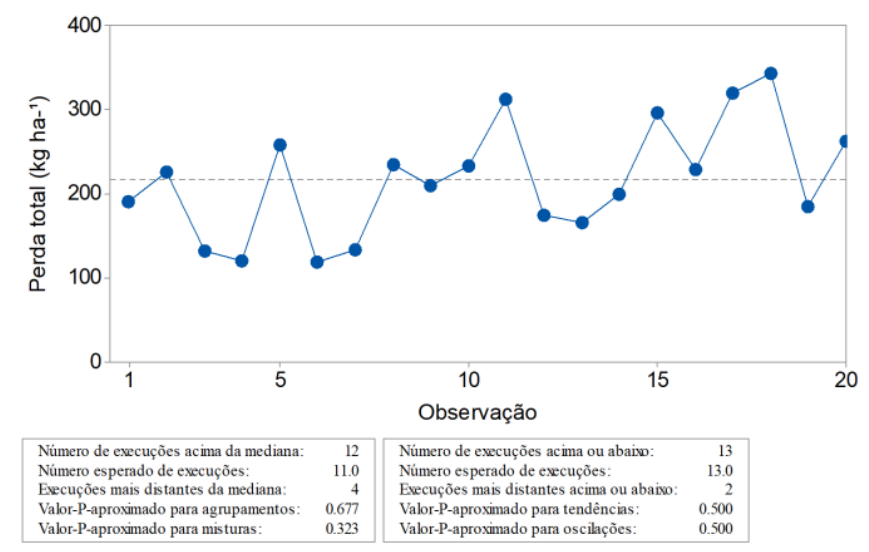

Figura 2. Gráfico sequencial para perdas totais $\left(\mathrm{kg} \mathrm{ha}^{-1}\right)$.

Quando a presença desses tipos de padrões é preciso adoção de medidas corretivas para que quando há detecção de causas especiais nas cartas de controle bem como padrões de não aleatoriedade nas run charts é recomendado corrigir as falhas, que são as causas especiais, o mais rápido possível pois quanto mais tempo demorar para reduzir essa falha na operação pode haver o aumento de perdas e por consequência diminuição da produtividade.

Normalmente essas causas especiais estão relacionadas com os fatores que afetam na produtividade bem como nas perdas como foi verificado por Silva (2019) esses fatores podem ser: excesso de maturação, doenças diversas, solo muito seco, compactado ou com baixa fertilidade, além da correta regulagem e manutenção das máquinas para colheita. Pela análise descritiva, na Tabela 1, foi observado que as medidas de tendência central, média e mediana, tiveram valores próximos, indicando uma possível distribuição normal e que foi constatado pelo teste de normalidade por Ryan-Joiner que os dados apresentaram distribuição normal. 
Para as medidas dispersão, desvio-padrão e coeficiente de variação, os dados não ficaram muito afastados da média e que embora ter sido considerado alto conforme a classificação de PimentelGomes e Garcia (2002), quando comparado com os outros trabalhos com colheita mecanizada, o valor obtido neste trabalho foi baixo, como os de Barrozo (2009) em amendoim, Menezes et al. (2018) em soja, Voltarelli et al. (2015) em cana-de-açúcar, e dentre outros. Quanto ao coeficiente de assimetria demonstrou distribuição assimétrica a direita (Ck > 0). E o coeficiente de curtose teve distribuição platicúrtica (Ck < 0) com uma curva mais achatada devido a dispersão dos dados em relação a medida central, a média.

Tabela 1. Análise descritiva para o indicador de qualidade perda total $\left(\mathrm{kg} \mathrm{ha}^{-1}\right)$ com a armação retangular para a cultura do amendoim.

\begin{tabular}{|c|c|c|c|c|c|c|c|}
\hline $\begin{array}{l}\text { Indicador } \\
\text { de } \\
\text { qualidade }\end{array}$ & $\mathbf{M}$ & Med & CV & $S$ & Cs & Ck & RJ \\
\hline $\begin{array}{l}\text { Perda } \\
\text { Total }\end{array}$ & 216,90 & 217,20 & 31,09 & 67,40 & 0,25 & -0.77 & $0,99^{\mathrm{N}}$ \\
\hline \multicolumn{8}{|c|}{$\begin{array}{l}\text { M: Média aritmética simples; Med: Mediana; CV: Coeficiente de Variação; S: } \\
\text { Desvio-padrão; Cs: Coeficiente de assimetria; Ck: Coeficiente de curtose; RJ: } \\
\text { Teste de normalidade por Ryan-Joiner; P: de acordo com o teste de Ryan- } \\
\text { Joiner test (N: Distribuição normal; A: Distribuição não normal com p-valor } \\
0.10 \text { ) }\end{array}$} \\
\hline
\end{tabular}

\section{Conclusões}

Concluiu-se que por meio da aplicação das ferramentas de qualidade permitiu o monitoramento da operação, em que não houve presença de causas especiais e nem de padrões de não aleatoriedade. Se caso tivesse sido detectado algum tipo de falha ao decorrer do recolhimento, seria recomendado a adoção de medidas corretivas para melhoria da qualidade.

\section{Agradecimentos}

A Coordenação de Aperfeiçoamento de Pessoal de Nível Superior - Brasil (CAPES) - Código de Financiamento 001, pela concessão da bolsa de mestrado do primeiro autor. 


\section{Referências}

ALENCAR, João Rui B. et al. Uso de Controle Estatístico de Processo para Avaliação da Estabilidade e Validação da Fase de Compressão de Formas Farmacêuticas Sólidas. Acta Farmacéutica Bonaerense, Buenos Aires, v. 24, n. 3, p. 426-435, 2005.

ALVARES, Clayton Alacarde et al. Köppen's climate classification map for Brazil. Meteorologische Zeitschrift, Stuttgart, v. 22, n. 6, p.711728, 2013. https://doi.org/10.1127/0941-2948/2013/0507

BARROZO, Leandra Matos. Perdas quali-quantitativas na colheita mecanizada de sementes de amendoim (Arachis hypogaea L.). 2009. 84 f. Dissertação (Mestrado) - Curso de Agronomia (Produção e Tecnologia de Sementes), Universidade Estadual Paulista (Unesp), Faculdade de Ciências Agrárias e Veterinárias, Jaboticabal, Jaboticabal, 2009.

MENEZES, Patricia Candida de et al. Can combine headers and travel speeds affect the quality of soybean harvesting operations? Revista Brasileira de Engenharia Agrícola e Ambiental, Campina Grande, v. 22 , n. 10, p. 732-738, out. 2018. http://dx.doi.org/10.1590/18071929/agriambi.v22n10p732-738

MONTGOMERY, Douglas C. Introdução ao controle estatístico da qualidade. 7. ed. Rio de Janeiro: LTC, 2016. 549 p.

PIMENTEL-GOMES, Frederico; GARCIA, Carlos Henrique. Estatística aplicada a experimentos agronômicos e florestais: exposição com exemplos e orientações para uso de aplicativos. Piracicaba: Fealq, 2002.

SANTOS, Edna. Estudo mapeia áreas de produção de amendoim do Brasil para prevenir doença do carvão. EMBRAPA - Empresa Brasileira de Pesquisa Agropecuária, 22 jan. 2019. Disponível em: https://www.embrapa.br/busca-de-noticias/-/noticia/40697528/estudomapeia-areas-de-producao-de-amendoim-do-brasil-para-prevenirdoenca-do-carvao. Acesso em: 22 jul. 2020.

SILVA, Rouverson Pereira da. Colheita mecanizada de amendoim. In: SILVA, Rouverson Pereira da; SANTOS, Adão Felipe dos; CARREGA, Willians César (ed.). Avanços na produção de amendoim. Jaboticabal: Funep, 2019. p. 129-146.

VOLTARELLI, Murilo Aparecido et al. Monitoring of mechanical sugarcane harvesting through control charts. Engenharia Agrícola, Jaboticabal, v. 35, n. 6, p. 1079-1092, dez. 2015. http://dx.doi.org/10.1590/1809-4430-eng.agric.v35n6p1079-1092/2015 